\title{
Supramolecular Complexes of Phospholipids and $\beta$-Cyclodextrin with Bioactive $\beta$-Carotene: A Comparative Physico-Chemical and Functional Evaluation
}

\author{
Saurabh Gujar ${ }^{1}$, Darshan Telange ${ }^{2}$, Anil Pethe ${ }^{3^{*}}$ \\ 1Shobhaben Pratapbhai Patel School of Pharmacy and Technology Management, SVKM's, NMIMS (Deemed to be University), \\ Mumbai Campus, Mumbai, Maharashtra, INDIA. \\ 2Department of Pharmaceutics and Nanotechnology, Rajarshi Shahu College of Pharmacy, Buldhana, Maharashtra, INDIA. \\ ${ }^{3}$ School of Pharmacy and Technology Management, SVKM's, NMIMS (Deemed to be University), Hyderabad Campus, Hyderabad, \\ Telangana, INDIA.
}

\begin{abstract}
Background: $\beta$-carotene, a chief component of carotenoids family exhibits multiple numbers of pharmacological activities. However, its poor aqueous solubility and low dissolution rate restricts it to become a potential drug candidate. Hence, $\beta$-carotenephospholipids complex (BPLC) and $\beta$-carotene- $\beta$-cyclodextrin complex (BCDC) were prepared with an objective of enhancing its aqueous solubility and dissolution rate. Materials and Methods: BPLC and BCDC were synthesized using solvent evaporation and kneading method respectively. BPLC and BCDC, were characterized by particle size and zeta potential analysis, complexation rate, drug loading, Fourier transform infrared spectroscopy and differential scanning calorimetry. Functional characterization of above formulations was performed by solubility and in vitro dissolution studies. Results and Conclusion: Particle size analysis result of BCDC and BPLC formulations were found to be suitable for oral route of administration. FT- IR and DSC studies supported the formation of BCDC and BPLC formulation. Solubility results displayed that BPLC (1:1) significantly enhanced the aqueous solubility upto (28-fold), compared to BCDC (1:2) (18-fold) and $\beta$-carotene. Dissolution studies showed that BPLC $(1: 1)$ considerably improved the release rate of $\beta$-carotene in PBS $(\mathrm{pH} 7.4)$ compared to $\operatorname{BCDC}(1: 2)$ and $\beta$-carotene suspension. Hence, above comparison confirmed that phospholipids could be promising carrier compared to $\beta$-cyclodextrin for overall enhancement of aqueous solubility and in vitro dissolution rate of $\beta$-carotene.
\end{abstract}

Key words: $\beta$-carotene, Phospholipids, $\beta$-cyclodextrin, Solubility, in vitro dissolution.

\section{INTRODUCTION}

$\beta$-carotene ( $\beta$-CTE), a chief component of carotenoids family shows existence in many fruits, vegetables and microalgae such as mangos, cantaloupe, peppers, pumpkin, sweet potatoes, carrots, leaves, fish and sea foods etc. It acts as a great source of vitamin $\mathrm{A}$ and hence, it finds application in the maintenance of normal functioning of human eye. ${ }^{1}$ Moreover, it is an approved food ingredients and thus, has been employed majorly in countless food, cosmetic and most importantly, in pharmaceutical products. ${ }^{2}$ Earlier studies have reported a number of clinical benefits of $\beta$-CTE such as antioxidant, ${ }^{3}$ anticancer, cardio-protective and anti-ageing activities. ${ }^{4,5}$ Regardless of therapeutic advantages of $\beta$-CTE, its clinical utility is primarily restricted by its poor aqueous solubility $(<0.6 \mu \mathrm{g} / \mathrm{mL})$, low oral bioavailability $(\sim 11-30 \%)$ and easy susceptibility to degradation due to high sensitivity to molecular oxygen, light and temperature. In this situation, overcoming
Submission Date: 22-11-2019; Revision Date: 06-02-2020; Accepted Date: 02-05-2020

DOI: 10.5530/ijper.54.2s.78 Correspondence: Dr. Anil M. Pethe School of Pharmacy and Technology Management SVKM's NMIMS (Deemed to be University), Polepally SEZ, TSIIC, Jadcherla, Mehbubnagar-509301, Hyderabad, Telangana, INDIA.

Phone: +91 8879212188 E-mail: anilpethe@gmail. com

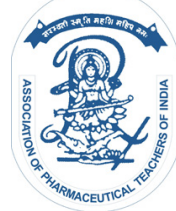

www.ijper.org 
of $\beta$-CTE limitations by using suitable carriers and/or methods could be more valuable.

Previously several reports demonstrated the turnaround the above limitations of $\beta$-CTE by employing several formulation strategies such as nanodispersion, ${ }^{6}$ nanoemulsion, $^{3,7}$ complexation $^{8}$ and solid dispersion. ${ }^{9}$ However, these formulations reported only the improvement of physical and chemical stability of $\beta$-CTE without investigating its aqueous solubility and in vitro dissolution rate. Improvement in these two parameters can provide more benefits in the processing of food products. Thus, there is a strong need to develop unique formulation using suitable carrier, which can overcome the observed significant limitations of $\beta$-CTE.

Among all existed formulations, complexation system is reported to be a most promising technique for the enhancement of poor aqueous solubility, in vitro dissolution rate, oral bioavailability and antioxidant activity of drug molecules. ${ }^{10,11}$ Carriers such as phospholipids and $\beta$-cyclodextrin are specifically utilized for the development and preparation of complexation system. Recent studies have evidenced that these carriers on complexation with plant bioactives such as salvianolic acid B, ${ }^{12}$ chlorogenic acid, ${ }^{13}$ ferulic acid $^{14}$ and idebenone $e^{15}$ appreciably enhanced the solubility, bioavailability and respective pharmacological activities. Complexation via phospholipids can form stable amorphous phospholipids complex, where bioactive get entrapped within the core of phospholipids and thus, form supramolecular complex by formation of $\mathrm{H}$ - bonds, van der Waals and ion-dipole forces between them. Amphiphilic and biocompatible nature of phospholipids can also act as preferable carrier for transportation of hydrophobic drug across biological membrane, which in turn, enhances the bioavailability of drugs. ${ }^{16}$ Due to its high amorphous characteristics, it significantly improves the aqueous solubility of hydrophobic drug via reducing its crystalline property and thereby, increases its wetting in aqueous media. ${ }^{17}$ Likewise, $\beta$-cyclodextrin $(\beta-C D)$ based complexation system is also called as inclusion complex. Due to its biocompatibility and superficial functionalization, it can freely occupy the varying size of hydrophobic drug within its central cavity (made up of 7-glucopyranose units), which may cause to dehydrate and transfer drug into cavity, form spatial arrangement inclusion complex via non-covalent interactions, resulting to increase the affinity of drug towards aqueous media with random transformation from crystalline to high energy state amorphous powder and finally improves the solubility of drugs. Additionally, the reported amphiphilic nature of $\beta$-CD molecule significantly improves the solubility and dissolution rate of hydrophobic drug via reducing the interfacial tension between the solid particles and dissolution medium. ${ }^{18}$ Moreover, both carriers provide multiple advantages to the formulation such as easy preparation process, high drug loading capacity and long term stability etc.

The present research work deals with an enhancement of poor aqueous solubility and dissolution rate of $\beta$-CTE by preparing its complex with phospholipids or $\beta-C D$ and evaluate them comparatively. BCDC and BPLC were prepared by kneading and solvent evaporation method. Prepared complex was physicochemically and functionally evaluated using particle size analysis, drug loading, FT-IR, DSC, solubility and in vitro dissolution rate.

\section{MATERIALS AND METHODS}

\section{Materials}

Lyophilizer (Model: MSW-137, Macro Scientific Works Pvt. Ltd., New Delhi, India). $\beta$-carotene (purity > 99\%) was obtained from Natural Remedies Pvt. Ltd., Bangalore, India. Cholesterol was received as gift sample from VAV Life Sciences Pvt. Ltd., Mumbai, India. Phospholipon ${ }^{\circledR} 90 \mathrm{H}$ was purchased from Molychem, Mumbai India. Absolute ethanol, ethylenediamine tetraacetic acid (EDTA), disodium hydrogen phosphate, potassium dihydrogen phosphate and sodium chloride were obtained from Loba Chemicals Pvt. Ltd., Mumbai, India. All other chemicals received for the study were of analytical grade reagent.

\section{Preparation of $\beta$-carotene $-\beta-$ cyclodextrin complex (BCDC)}

The $\beta$-cyclodextrin based complex of $\beta$-carotene was prepared in molar ratios of (i.e. 1:1, 1:2 and 1:3) using kneading method reported earlier in literature. ${ }^{19}$ Briefly, the $\beta$-CTE and $\beta$-CD, both were accurately and individually weighed according to their molar ratios and then transferred into a clean and previously air-dried mortar. The weighed mixture was mixed and dissolved using $20 \mathrm{~mL}$ of absolute ethanol. The solvent was allowed to evaporate, which in turn, resulting to formation of porous solid mass. The obtained mass was dried using hot air oven at $50^{\circ} \mathrm{C}$ for $24 \mathrm{hr}$ for removal of entrapped solvent, if any. The dried mass was sieved to get uniform size particles. These particles were then placed in an amber colored glass vials previously flushed with nitrogen and finally stored at RT, till further physico-chemical analysis. 


\section{Preparation of $\beta$-carotene- phospholipids complex (BPLC)}

Thin film hydration technique was employed for the preparation of $\beta$-CTE - phospholipids complex with slight modifications. ${ }^{20}$ Briefly, as per above mentioned molar ratios, the same quantities of $\beta$-CTE and Phospholipon ${ }^{\circledR} 90 \mathrm{H}$ were individually weighed and transferred into $250 \mathrm{~mL}$ round bottom flask. The powdered samples were co-dissolved in $10 \mathrm{~mL}$ of absolute ethanol. The prepared solution was magnetically stirred (at $25^{\circ} \mathrm{C}$ for $1 \mathrm{hr}$ ) to ensure uniform mixing of both the components. After stirring, the assembly was subjected to rotary vacuum evaporator, leading to evaporation of solvent and formation of film. The obtained film was hydrated using $5 \mathrm{~mL}$ of deionized water and then sonicated. The hydrated solution was then lyophilized using a lyophilizer (Model: MSW-137, Macro Scientific Works Pvt. Ltd., New Delhi, India) under the conditions of controlled condenser temperature of $-80^{\circ} \mathrm{C}$ and vacuum of $10 \mathrm{~Pa}$. The final dried BPLC was preserved in nitrogen flushed and light resistant container and stored at $4^{\circ} \mathrm{C}$ for further analysis. Composition of BCDC and BPLC formulation are shown in Table 1.

\section{Estimation of drug loading and extent of complexation rate (\% yield)}

The inclusion of $\beta$-CTE within the prepared BCDC was estimated by well-known spectrophotometric method described earlier in the literature. ${ }^{21}$ Briefly, the prepared BCDC formulations was accurately weighed (equivalent to $\sim 10 \mathrm{mg}$ of pure $\beta$-CTE) and mixed with $100 \mathrm{~mL}$ of phosphate buffer solution (0.05M, $\mathrm{pH} 6.8$ ). The prepared solution was then subjected to membrane filtration $(0.45 \mu)$ in order to get clear filtrate. From this solution, few aliquots were removed, diluted appropriately, analyzed for absorbance at suitable detection wavelength $\left(\lambda_{\max }=450 \mathrm{~nm}\right)$ on UV-visible

\begin{tabular}{|c|c|c|}
\hline Formulations & $\beta$-carotene (mg) & $\beta$-cyclodextrin (mg) \\
\hline \multicolumn{3}{|c|}{ BCDC } \\
\hline BCDC 1 & 536.88 & 1135 \\
\hline BCDC 2 & 536.88 & 2270 \\
\hline BCDC 3 & 536.88 & 3405 \\
\hline Formulations & $\beta$-carotene $(\mathrm{mg})$ & Phospholipon $^{\circledast}$ 90H (mg) \\
\hline \multicolumn{3}{|c|}{ BPLC } \\
\hline BPLC 1 & 536.88 & 790 \\
\hline BPLC 2 & 536.88 & 1580 \\
\hline BPLC 3 & 536.88 & 2370 \\
\hline
\end{tabular}

spectrophotometer (Model: V-630, JASCO International Co., Ltd., Tokyo, Japan). The separate carrier solution with same concentration was also prepared to remove any developed interference while performing analysis. A method previously described by Tan $e t$ al. ${ }^{22}$ was used for the determination of extent of $\beta$-CTE in the prepared BPLC formulations. Briefly, the chloroform based dispersion was prepared by dissolving BPLC (equivalent to $\sim 50 \mathrm{mg}$ of $\beta$-CTE) into $5 \mathrm{~mL}$ of chloroform, followed by stirring, resulting to formation of uniform dispersion. In this dispersion, the BPLC and Phospholipon ${ }^{\circledR} 90 \mathrm{H}$ were showed complete solubility, whereas, pure $\beta$-CTE was remained undissolved and settled down at the bottom of the beaker. Next, the dispersion was filtered using Whatman ${ }^{\circledR}$ filter paper (ashless, Grade 41, Sigma-Aldrich Corporation, St. Louis, MO) and separate the undissolved residue. This residue was dried at room temperature $\left(25^{\circ} \mathrm{C}\right)$. After drying, it was then dissolved in methanol, diluted suitably and analyzed the resulting solution on UV-visible spectrophotometer (Model: V-630, JASCO International Co., Ltd., Tokyo, Japan) at detection wavelength of $\left(\lambda_{\max }=450 \mathrm{~nm}\right)$. The below described equation (1) was used to calculate the extent of complexation rate of pure $\beta$-CTE with Phospholipon ${ }^{\circledR}$ 90H.

Extent of complexation $(\%)=\left(c_{t}-c_{f}\right) / c_{t} \times 100$

Where, $c$ represents the theoretical concentration of $\beta$-CTE in BPLC and $c$ designates the observed concentration of $\beta$-CTE in the filtrate.

\section{Physico-chemical characterization}

\section{Particle size and zeta potential}

A well established Photon Cross-Correlation Spectroscopy (PCCS) attached with Dynamic Light Scattering (DLS) technology used by our laboratory previously, was utilized for determination of particle size distribution and zeta potential of BCDC and BPLC formulations. ${ }^{23}$ Briefly, BCDC and BPLC formulations (equivalent to $\sim 5 \mathrm{mg}$ ) was weighed, dispersed in $10 \mathrm{~mL}$ of deionized water and stirred well. The prepared dispersion was placed into sample holder and examined in the sensitivity range of $1 \mathrm{~nm}$ to $10 \mu \mathrm{m}$ using particle size analyzer (Model: NANOPHOX Sympatec, GmbH, Clausthal-Zellerfeld, Germany). The analyzer analyzed the particle size distribution of both formulations by optimization of particle count rate via adjusting the sample position. The analysis was carried out at room temperature $\left(25^{\circ} \mathrm{C}\right)$. Obtained results were read by using software accompanying with this instrument. 
The aqueous dispersion of BCDC and BPLC as prepared above, was also used for the determination of zeta potential within the sensitivity range of -200 to $+200 \mathrm{mV}$ using Nano Particle Analyzer (Model: NanoPlusTM-2, Particulate system, Norcross, GA, USA) attached with Dynamic Light Scattering (DLS) technology. This operational procedure was carried out at RT.

\section{FT-IR study}

The molecular level interactions between the samples of $\beta$-CTE, $\beta$-CD, Phospholipon ${ }^{\circledR} 90 \mathrm{H}$, physical mixture $(\mathrm{PM})$ of $\beta$-CTE and $\beta$-CD, PM of $\beta$-CTE and Phospholipon ${ }^{\circledR} 90 \mathrm{H}, \mathrm{BCDC}$ and BPLC formulations were analyzed using FT-IR spectrophotometer (Model: FTIR-8300, Shimadzu, Kyoto, Japan) procedure previously reported by our members. ${ }^{24}$ Briefly, an approximate amount of each sample $(\sim 2 \mathrm{mg})$ and FT-IR grade of potassium bromide $(\sim 200 \mathrm{mg})$ were weighed, transferred and mixed well. The prepared homogenous powder were collected and then compressed into transparent discs using Mini-Hand Press Machine (Model: MHP-1, $\mathrm{P} / \mathrm{N}-200-66747-91$, Shimadzu, Kyoto, Japan) at a pressure of 10 ton $/ \mathrm{Nm}^{2}$. Each sample discs were scanned (32 scans) within the wave number range of (4000 to $400 \mathrm{~cm}^{-1}$ ) with scanning resolution was fixed at $4 \mathrm{~cm}^{-1}$. After this, the scanned FT-IR absorption peaks for each sample were analyzed and interpreted by software (IR solution, Version 1.10) accompanying with this instrument.

\section{DSC study}

A differential scanning calorimeter (Model: DSC-1 821e, Mettler-Taledo AG, Analytical, Schwerzenbach, Switzerland) was employed to understand the thermal characteristics of above mentioned components of BCDC and BPLC formulations. A DSC analysis procedure earlier reported by our group was employed in this study. ${ }^{25}$ Briefly, the formulation components were individually weighed $(\sim 2.0 \mathrm{mg} \pm 0.2)$ and transferred to the clean glass vials. Before analysis, the DSC instrument was calibrated by purified standard Indium (In) in order to get uniform heat flow and heat capacity. Sample analyzing area was also cleared with entrapped oxygen by purging liquid $\mathrm{N}_{2}$ at a flow rate of $50 \mathrm{~mL} / \mathrm{min}$. Weighed samples were then subjected to heating within the fixed range of 40 to $400^{\circ} \mathrm{C}$ or more, if any, at a temperature increment of $10^{\circ} \mathrm{C} / \mathrm{min}$. The final thermal spectrums of each component were read using the attached software (Universal Analysis 2000, V4.5A, Build 4.5.0.5) accompanying with instrument.

\section{Solubility studies}

The solubility performance of $\beta$-CTE, PM, BCDC and BPLC formulations in water and/or $n$-octanol were analyzed by following the procedure as described by Singh et al. ${ }^{17}$ Briefly, the aqueous and/or $n$-octanol dispersion (approximate $5 \mathrm{~mL}$ each) with an excess amount of each individual sample was prepared and then agitated on water bath shaker temperature (Model: RSB-12, Remi House, Mumbai, India) for $24 \mathrm{hr}$, leading to formation of homogenous dispersion. The prepared dispersion was centrifuged at 1500 RPM for $25 \mathrm{~min}$ and filtered using $0.45 \mu$ membrane. From the obtained filtrate, the small aliquots was pipette out, diluted suitably and analyzed the resulting solution at maximum wavelength of $\left(\lambda_{\max }=450 \mathrm{~nm}\right)$ using UV-visible spectrophotometer (Model: V-630, JASCO International Co., Ltd., Tokyo, Japan). This analytical procedure was performed at room temperature $\left(25^{\circ} \mathrm{C}\right)$.

\section{Functional characterization}

\section{In vitro dissolution studies}

In this study, the dialysis membrane dissolution method, earlier described by Maiti et al. ${ }^{26}$ was used to study the comparative in vitro dissolution performance of $\beta$-CTE, BCDC and BPLC formulations. Briefly, the dialysis membrane with following characteristics such as (LA395, dialysis membrane-110, average diameter $\sim 21.34$, average flat width $\sim 32$, capacity $\sim 3.63 \mathrm{~mL}$, molecular size cut-off range 12,000 - 14,000 Da, HiMedia Laboratories, Mumbai, India) was employed in the current study. The procured membrane was washed and rinsed as per the suggestions received from the manufacturer. After washing, the small size dialysis bags were prepared, loaded with samples such as $\beta$-CTE $(\sim 2 \mathrm{mg})$, BCDC $(\sim 2 \mathrm{mg}$ of pure $\beta$-CTE) and BPLC formulations ( $\sim 2 \mathrm{mg}$ of pure $\beta$-CTE) and then tied using thread. Moreover, bags loaded with samples were also checked for leakage, if any. Next to this, the bags containing samples were suspended in vertical position into freshly prepared phosphate buffered-saline (PBS, $200 \mathrm{~mL}, \mathrm{pH}$ 7.4) with Tween ${ }^{\circledR} 20$ (1\%, v/v) solution dissolution media and stirred well at 50 RPM using magnetic stirrer. The small solution was removed at a fixed time intervals, diluted appropriately and assayed at maximum wavelength $\left(\lambda_{\max }=450 \mathrm{~nm}\right)$ for recording the absorbance on UV-visible spectrophotometer (Model: V-630, JASCO International Co., Ltd., Tokyo, Japan). The measured absorbance gives the information regarding cumulative release of $\beta$-CTE from its suspension, BCDC and BPLC over the period of $12 \mathrm{hr}$. 


\section{RESULTS AND DISCUSSION}

\section{Formulation of BCDC and BPLC}

The oral bioavailability of drug mainly depends upon the numerous physical properties of drug such as low aqueous solubility, permeability, dissolution rate, first-pass metabolism and presystemic metabolism. Among all, the poor aqueous solubility and low permeability are the main properties of drugs which can influence the poor oral bioavailability. ${ }^{27} \beta$-CTE, an investigational drug candidate, also demonstrates poor aqueous solubility and fair solubility in most of the organic solvents. ${ }^{28}$ According to this physical property, $\beta$-CTE was processed into BCDC and BPLC formulations via kneading and thin film hydration method respectively. Many stable formulations of $\beta-C D$ and phospholipids-based complexes with flavonoids/ terpenoids have been successfully developed by authors with the use of dichloromethane, ${ }^{29}$ water and methanol $(1: 1),{ }^{19} 1$, 4-dioxane ${ }^{23}$ and tetrahydrofuran (THF). ${ }^{30}$ In the current study, we have adopted the same formulation strategy as well as solvents for the preparation of flavonoids/terpenoids - $\beta$-CD as well as phospholipids complex. However, during processing, the formulation components showed an insolubility and/or poor solubility in these solvents, which in turn, resulting to precipitation of $\beta$-CTE, $\beta$-CD and phospholipids respectively. Poor solubility problem of these formulation components was solved by employing the new series of solvents. From all these investigated solvents, the absolute ethanol with its semi-polar nature, class III solvents and low toxic nature exhibited a preferable solvent of choice for the rapid dissolution of $\beta$-CTE, $\beta$-CD and phospholipids respectively. Therefore, the absolute ethanol was chosen as a solvent of choice for the preparation of BCDC and BPLC formulations.

\section{Drug loading and extent of complexation rate (\% yield)}

Results of drug loading and extent of complexation rate for BCDC and BPLC formulations are discussed below. The values of drug loading and extent of complexation rate for all BCDC and BPLC formulations were found to be in the range of 91 to $94 \% \mathrm{w} / \mathrm{w}$ and 96 to $98 \% \mathrm{w} / \mathrm{w}$, respectively. The BCDC 2 (ratio 1:2) and BPLC 1 (ratio 1:1) formulation showed higher value around $\sim 97.60 \pm 0.20 \%$ and $\sim 98.33 \pm 0.18 \%$ respectively. Moreover, the obtained higher values of BCDC 2 and BPLC 1 formulations were selected as optimized formulations for further characterization. It was found that adopted method used for the estimation of drug loading and complexation rate is robust, validated and clearly supports the selected and incorporated carrier.

\section{Particle size and zeta potential analysis}

The physical stability of sub-micron particles that dispersed in the liquid medium is assessed by estimation of its mean particle size and zeta potential respectively. The analyzed mean particle size distribution of BCDC and BPLC formulations are shown in Figure 1 (a and c). Stavic et al. have reported that particle size $<500 \mathrm{~nm}$ particularly, used endocytosis pathway for its transportation across the biological membrane. ${ }^{31}$ In this study, BCDC formulation showed mean particle size and polydispersity index (PDI) around $\sim 162 \mathrm{~nm}$ and $\sim 0.27 \pm 0.09$, whereas, the mean particle size and PDI values for BPLC formulations was found to be $\sim 119.12$ $\mathrm{nm}$ and $\sim 0.35 \pm 0.08$ respectively. Results for BCDC and BPLC indicates that its suitability for oral route of administration with wide range of particle distribution. These findings were in agreement with earlier published reports. ${ }^{32}$ In addition to particle size, the zeta potential $(\zeta)$ is another valuable stability indicator, used for the determination of charges on the surface of the particles and also possibly helps in determination of its performance upon oral administration. The acceptable range of zeta potential values for multiparticulate system is reported to be in between $-30 \mathrm{mV}$ to $+30 \mathrm{mV}$ respectively. Figure $1(\mathrm{~b}$ and $\mathrm{d}$ ) shows the zeta potential values of BCDC and BPLC formulations. The zeta potential values for BCDC and BPLC were found about $\sim-10.7 \pm 0.15 \mathrm{mV}$ and $-6.50 \pm 0.10 \mathrm{mV}$ respectively. From the combined results it indicates that BCDC and BPLC, both shows the desirable physical stability and it was attributed to lower particle size, zeta potential and higher PDI value.

\section{FT-IR study}

FT-IR spectrum of $\beta$-CTE, $\beta$-CD, Phospholipon ${ }^{\circledR} 90 \mathrm{H}$, BCDC and BPLC formulations are shown in Figure 2

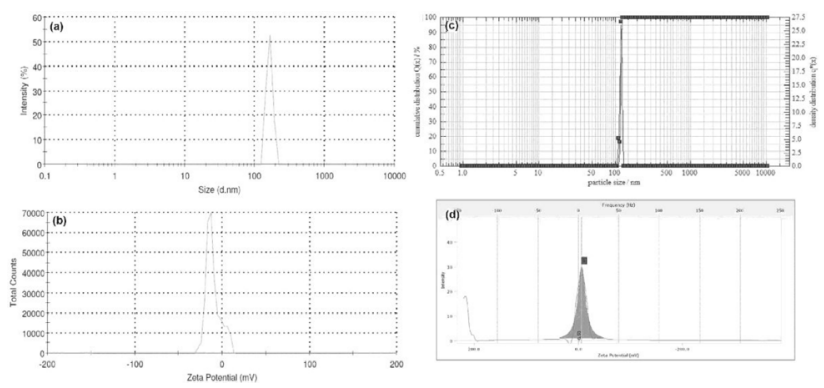

Figure 1: Particle size distribution of (a) BCDC and (c) BPLC, formulations and zeta potential analysis of (b) BCDC and (d) BPLC formulations. 


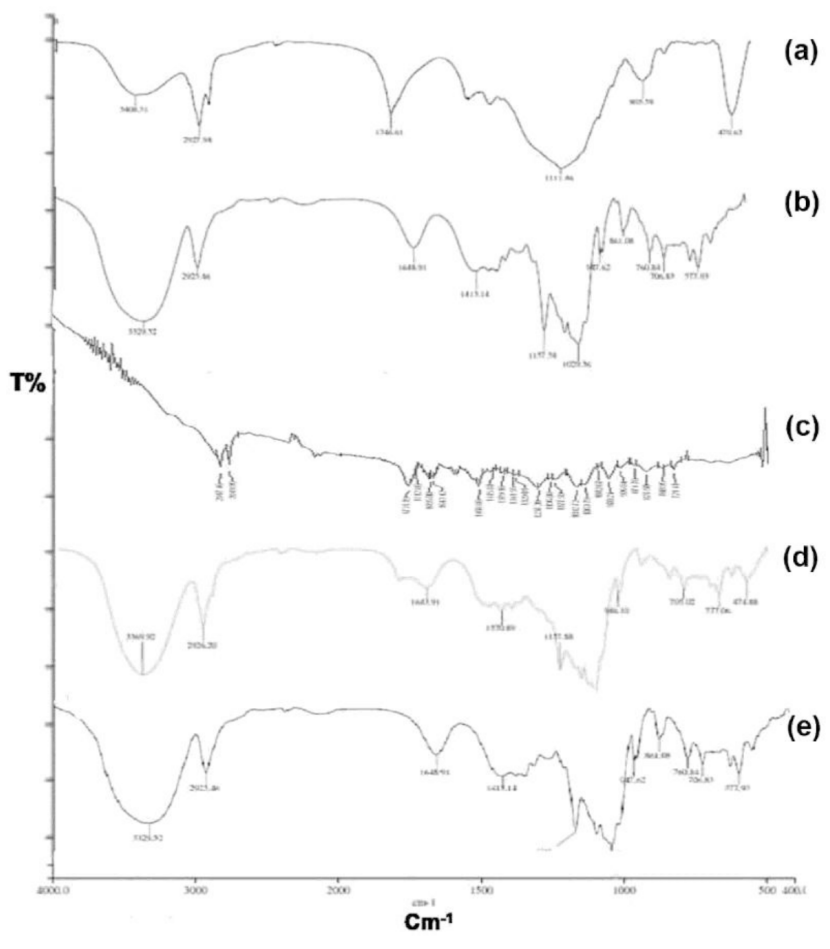

Figure 2: FT-IR spectrum of (a) $\beta$-CTE, (b) $\beta-C D$, (c) Phospholipon ${ }^{\circledR 90 H}$, (d) BCDC, (e) BPLC formulations.

(a, b, c, d and e) respectively. The units are shown in $\mathrm{cm}^{-1}$. $\beta$-CTE (Figure 2a) showed absorption peak at 2927 for asymmetric (aliphatic C-H stretching) and 2874 for symmetric (aromatic C-H stretching). Absorption peak at 1458 and 1360 shows the presence of aromatic (C-C stretching) and symmetric (C-H stretching) group. Peak observed at 1746 and 803 corresponds to carbonyl group stretching and (= $\mathrm{CH}$ group) in alkenes. ${ }^{33}$ FT-IR spectrum of $\beta-C D$ is shown in (Figure $2 b$ ). Observed absorption peak at 3329, 2952, 1413 and 1029 indicates the presence of (O-H stretching), aliphatic ( $\mathrm{C}-\mathrm{H}_{2}$ bending), (aliphatic C-H stretching) and (C-O stretching) vibration groups respectively. These peaks were found in agreement with earlier reports. ${ }^{29}$ (Figure 2c) shows the absorption peaks of Phospholipon ${ }^{\circledR} 90 \mathrm{H}$. Peak found at 2917 and 2849 represents the presence of (C-H stretching) of long fatty acid chain. Additional peaks were observed at 1731, 1237 and 1092 and 970 for $(\mathrm{C}=\mathrm{O}$ stretching vibration for fatty acid ester), ( $\mathrm{P}=\mathrm{O}$ and $\mathrm{P}-\mathrm{O}-\mathrm{C}$ stretching) and $\left[-\mathrm{N}^{+}\left(\mathrm{CH}_{2}\right) 3\right] .{ }^{17}$ FT-IR spectrum of BCDC in (Figure 2d) exhibited peaks shifted at 3369, 2926 and 1643 compared to that of plain $\beta$-CTE and $\beta$-CD, whereas, in (Figure 2e), the BPLC also showed peaks shifted at 2925 and 1240 from their original peaks of $\beta$-CTE and Phospholipon ${ }^{\circledR} 90 \mathrm{H}$. Shifting of these peaks in BCDC as well as in BPLC formulation could be ascribed to strong participation of weak intermo- lecular interaction i.e. H-bonding, ion-dipole and van der Waals forces between polar part of $\beta$-CTE and carriers, which further resulting to formation of BCDC and BPLC.

\section{DSC study}

Figure $3 \mathrm{a}, 3 \mathrm{~b}, 3 \mathrm{c}, 3 \mathrm{~d}$ and $3 \mathrm{e}$ depicts the DSC thermograms of pure $\beta$-CTE, $\beta$-CD, Phospholipon ${ }^{\circledR} 90 \mathrm{H}$, BCDC and BPLC formulations. Pure $\beta$-CTE (Figure 3a) showed strong and sharp endothermic peak around $\sim 187.17^{\circ} \mathrm{C}$ indicates its purity and crystalline nature. ${ }^{9}$ A DSC curve of $\beta-C D$ is shown in (Figure $3 b$ ). It exhibits two endothermic peaks; first board peak appeared around $\sim 100^{\circ} \mathrm{C}$, indicative to release of water molecules from $\beta$-CD. Second board melting peak with less intensity were appeared above $\sim 300^{\circ} \mathrm{C}$, corresponds to its degradation peak. These results were found to be consistent with earlier published reports. ${ }^{34}$ As revealed in (Figure 3c), Phospholipon ${ }^{\circledR}$ $90 \mathrm{H}$ displays five different peaks around $\sim 83.62^{\circ} \mathrm{C}$, $105.11^{\circ} \mathrm{C}, 122.00^{\circ} \mathrm{C}, 178.20^{\circ} \mathrm{C}$ and $238.24^{\circ} \mathrm{C}$ respectively. First three peaks with small, diffused and mild characteristics were likely due to melting nature of Phospholipon $^{\circledR} 90 \mathrm{H}$ under the influence of increasing temperature. Last two small intensity peaks were ascribed by physical transformation of Phospholipon ${ }^{\circledR}$ $90 \mathrm{H}$ form gel to liquid crystal state via physicochemical modification in the carbon-hydrogen segment..$^{35}$ Two new endothermic peaks at about $\sim 73.26^{\circ} \mathrm{C}$ and

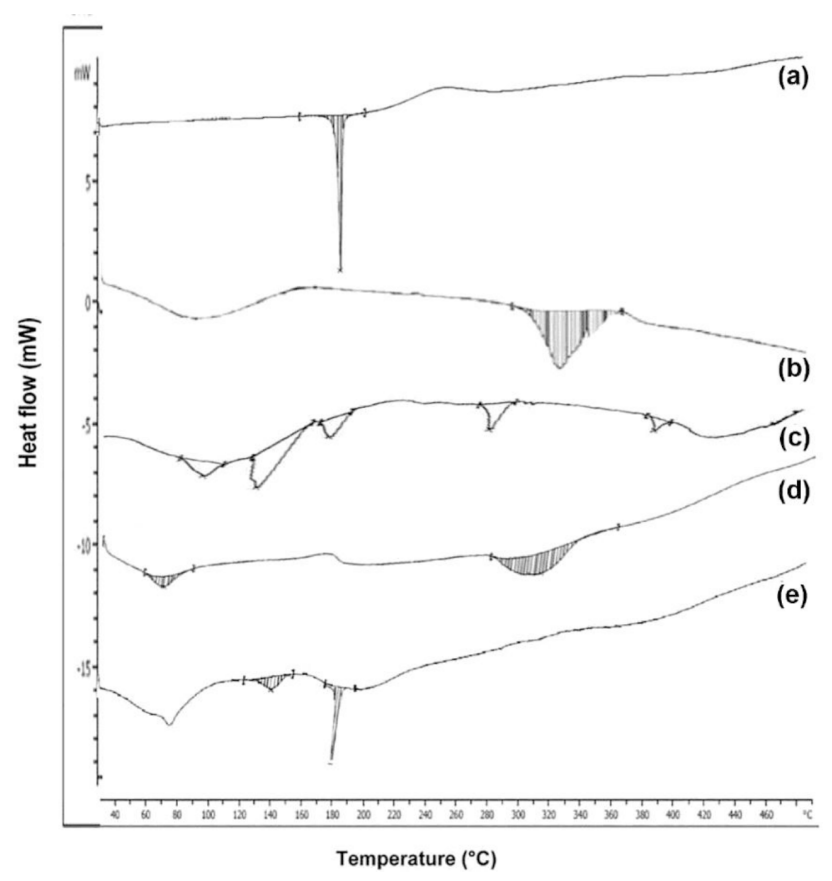

Figure 3: DSC thermograms of (a) $\beta-C T E$, (b) $\beta-C D$, (c) Phospholipon ${ }^{\circledR}$ OH, (d) BCDC, (e) BPLC formulations. 
$\sim 318.18^{\circ} \mathrm{C}$ with different intensities were displayed by BCDC formulations in (Figure 3d). First peak was appeared to be completely new one with disappearance of unusual peaks of $\beta$-CTE and $\beta$-CD. Moreover, the peak at $\sim 318.18^{\circ} \mathrm{C}$ represents the same feature as that of original second peak of $\beta-C D$ indicates that it didn't contribute in the complexation process. BPLC (Figure 3e) exhibited two new melting peaks around $\sim 147.29^{\circ} \mathrm{C}$ and $\sim 192.17^{\circ} \mathrm{C}$ respectively. Additionally, $\beta$-CTE and Phospholipon ${ }^{\circledR} 90 \mathrm{H}$ peak were absent in this thermograms. Weak forces of interactions such as ion-dipole and van der Waals forces between polar part of $\beta$-CTE and carriers could thus be the main reason for BCDC and BPLC synthesis. Findings are well supported by earlier published literature. ${ }^{36}$

\section{Solubility studies}

Solubility results of pure $\beta$-CTE, PM of $\beta$-CTE and $\beta$-CD (1:2), PM of $\beta$-CTE and Phospholipon ${ }^{\circledR} 90 \mathrm{H}$ (1:1), BCDC and BPLC formulations in water and/or $\mathrm{n}$-octanol are presented in Table 2. Pure $\beta$-CTE shows low aqueous solubility up to $\sim 0.52 \mu \mathrm{g} / \mathrm{mL}$, whereas, the same compound exhibited higher solubility in n-octanol i.e. $\sim 714.10 \mu \mathrm{g} / \mathrm{mL}$, indicates strong lipophilic and poor hydrophilic nature of $\beta$-CTE. PM of $\beta$-CTE and $\beta$-CD (1:2) as well as $\beta$-CTE and Phospholipon ${ }^{\circledR}$ $90 \mathrm{H}(1: 1)$ displays somewhat better aqueous solubility around $\sim 3.11 \mu \mathrm{g} / \mathrm{mL}$ (6-fold) and $\sim 4.61 \mu \mathrm{g} / \mathrm{mL}$ (9-fold) over to that of $\beta$-CD. The significance level was found to be $(p<0.05)$. Formation of a close association of $\beta$-CTE with $\beta$-CD and Phospholipon ${ }^{\circledR} 90 \mathrm{H}$ is thought to be main reason for slight improvement in the PM aqueous solubility. Both PM did not show any significant improvement in the n-octanol solubility. Compared to pure $\beta$-CTE and PM, the BCDC (1:2) offered a significant $(p<0.05)$ higher solubility in water around $\sim 9.21 \mu \mathrm{g} / \mathrm{mL}$ and it is enhanced by 18 -fold. This finding is well supported by earlier reports, which

Table 2: Solubility analysis of pure $\beta$-CTE, PM of $\beta-C T E$ and $\beta-C D$ and Phospholipon ${ }^{\circledR} 0 \mathrm{H}, \mathrm{BCDC}$ and BPLC formulations.

\begin{tabular}{|c|c|c|}
\hline Formulations & $\begin{array}{c}\text { Aqueous } \\
\text { solubility }(\boldsymbol{\mu g} / \mathrm{mL})\end{array}$ & $\begin{array}{c}\mathrm{n} \text {-octanol solubility } \\
(\boldsymbol{\mu g} / \mathrm{mL})\end{array}$ \\
\hline Pure $\beta$-CTE & $0.52 \pm 0.08$ & $714.10 \pm 1.32$ \\
\hline $\begin{array}{c}\text { PM of } \beta \text {-CTE and } \\
\beta \text {-CD }\end{array}$ & $3.11 \pm 0.24$ & $769.21 \pm 1.26$ \\
\hline $\begin{array}{c}\text { PM of } \beta \text {-CTE and } \\
\text { Phospholipon }{ }^{\circledast} 90 \mathrm{H}\end{array}$ & $4.61 \pm 0.19$ & $802.01 \pm 1.12$ \\
\hline BCDC 2 & $9.21 \pm 1.41$ & $872.43 \pm 1.48$ \\
\hline BPLC 1 & $14.67 \pm 1.38$ & $931.22 \pm 2.33$ \\
\hline
\end{tabular}

All results are expressed as mean \pm Std. Dev., $n=3$ suggest that central cavity of $\beta$-CD (made up of 7 -glucopyranose units) freely accommodates the $\beta$-CTE molecules, forms soluble inclusion complex with random transformation from crystalline to amorphous powder, resulting to improve aqueous solubility of $\beta$-CTE. ${ }^{37}$ The n-octanol solubility was also found to be higher $(\sim 872.49 \mu \mathrm{g} / \mathrm{mL})$ compared to pure drug and PM. Finally, BPLC (1:1) showed an excellent and more significant $(\phi<0.01)$ improved aqueous solubility around $\sim 14.61 \mu \mathrm{g} / \mathrm{mL}$ (28-fold), compared to drug, PM and BCDC formulations and it was likely due to partial amorphization and amphiphilic nature of BPLC. ${ }^{17}$

\section{In vitro dissolution studies}

The comparative in vitro dissolution performance of $\beta$-CTE from $\beta$-CTE suspension, BCDC (1:2) and BPLC (1:1) formulations in PBS ( $\mathrm{pH} 7.4$ ) for testing period up to $12 \mathrm{hr}$ are described in Figure 4. The release pattern of $\beta$-CTE from its suspension after $5 \mathrm{hr}$ was found to be $\sim 12 \%$ and by the end of $12 \mathrm{hr}$ dissolution period, the suspension showed only $\sim 20 \%$ of $\beta$-CTE released. This lowered amount of release could be attributed by poor aqueous solubility of $\beta$-CTE. Compared to suspension, the BCDC (1:2) formulation, after $5 \mathrm{hr}$ of testing period, released $\beta$-carotene around $~ 29 \%$, however, after this period, the BCDC demonstrated a continued release performance and thereby, reached to a maximum release about $\sim 68 \%$ by the end of dissolution period. Enhancement of dissolution rate of BCDC formulation could be well explained by favorable strong interaction between $\beta$-CTE and apolar cavity of $\beta-C D$, which may cause to dehydrate and

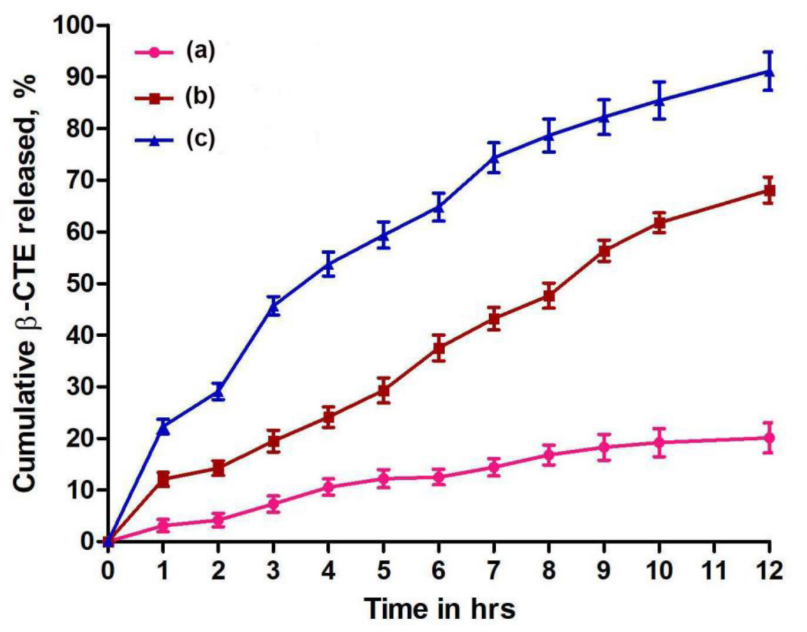

Figure 4: The in-vitro dissolution profiles of (a) $\beta$-CTE release from $\beta$-CTE suspension, (b) BCDC and (c) BPLC formulations. Values are presented as mean \pm Std. Dev. $(n=3)$. 
transfer the drug into the cavity, resulting to increase the affinity of hydrophobic drug towards the water and subsequently, increase the dissolution rate. Additionally, the reported amphiphilic nature of $\beta-C D$ molecule significantly improves the dissolution rate of hydrophobic drug via reducing the interfacial tension between the solid particles and dissolution medium. ${ }^{18,38}$ The rate and extent of dissolution of BPLC (1:1) formulation, after $5 \mathrm{hr}$ of study, was observed to be $\sim 59 \%$ and by the end of dissolution period, the same formulation continuously released the $\beta$-CTE to a maximum level about $\sim 91 \%$. This higher release was likely attributed to solubility and wettability phenomenon, where Phospholipon $^{\circledR} 90 \mathrm{H}$ on complexation with the $\beta$-CTE particles can increase its solubility and then wettability in water, forms uniform dispersion and consequently increase the dissolution rate. ${ }^{39}$ This comparison clearly indicates that BPLC formulation enhanced the dissolution of $\beta$-CTE more significantly over to that of plain drug and BCDC. After release study, the kinetic analysis of BCDC and BPLC formulations via examined different release models, exhibited higher correlation coefficient $\left(R^{2}\right)$ about $\sim 0.9943$ and $\sim 0.9889$ respectively and thereby, both of these followed the first order and Higuchi as best-fit dissolution mechanism. Moreover, the release exponent value $(n)$ of BCDC and BPLC demonstrated around $\sim 0.48$ and $\sim 0.49$ respectively. Based on these values, it was found that, BCDC and BPLC displayed the Fickian diffusion as the principal release mechanism. The improved solubility and dissolution rate of $\beta$-CTE using Phospholipon ${ }^{\circledR} 90 \mathrm{H}$ via thin film hydration technique could be helpful in the enhancement of its permeability and thereby improving the in vivo antioxidant, biopharmaceutical and most important, the pharmacokinetic potential.

\section{CONCLUSION}

The phospholipids and $\beta$-cyclodextrin carriers have shown the noteworthy potential for enhancement of aqueous solubility and in vitro dissolution rate of $\beta$-CTE. This goal was achieved by the preparation of BCDC and BPLC formulation using kneading and solvent evaporation method respectively. Based on lower particle size, zeta potential and PDI values, BCDC and BPLC both were found to show oral route suitability. Physico-chemical characterization by FT-IR and DSC studies showed that BCDC and BPLC, both were formed with the involvement of weak intermolecular forces of interactions such as H-bonding, ion-dipole and van der Waals forces between the polar part of $\beta$-CTE and carriers. BPLC (1:1) significantly enhanced the aqueous solubility around (28-fold), compared to that of BCDC (1:2) (18-fold) and pure $\beta$-CTE. Equally, rate and extent of dissolution of BPLC $(1: 1)$ was found to be enhanced more significantly over to that of BCDC (1:2) and pure $\beta$-CTE for $12 \mathrm{hr}$ testing period. In conclusion, the phospholipids carriers-based BPLC formulations could be employed as a promising carrier, compared to $\beta$-CD carrier for enhancement of poor aqueous solubility and in vitro dissolution rate of $\beta$-CTE and similar bioactive compounds. Moreover, the improved solubility and dissolution rate of $\beta$-CTE using thin film hydration method could be helpful in the enhancement of its permeability, in vivo antioxidant and pharmacokinetic potential.

\section{ACKNOWLEDGEMENT}

The corresponding author thanks to Dr. Bala Prabhakar, Dean, School of Pharmacy and Technology Management, SVKM's, NMIMS (Deemed to be University), Mumbai for providing necessary facilities to complete this research work.

\section{CONFLICT OF INTEREST}

The author declares no conflict of interest.

\section{ABBREVIATIONS}

$\beta$-carotene: $\beta$ - CTE; $\beta$-cyclodextrin: $\beta$-CD; BCDC: $\beta$-carotene- $\beta$-cyclodextrin complex; BPLC: $\beta$-carotenephospholipids complex; PM: Physical mixture; DSC: Differential scanning calorimetry; FT-IR: Fourier transform infrared spectroscopy.

\section{REFERENCES}

1. Boon C, McClements D, Weiss J, Decker A. Factors influencing the chemical stability of carotenoids in foods. Crtl Rev Food Sci and Nutr. 2010;50(6):515-32.

2. Martín A, Mattea F, Gutiérrez L, Miguel F, Cocero MJ. Co-precipitation of carotenoids and bio-polymers with the supercritical anti-solvent process. J of Supercritical Fluids. 2007;41(1):138-47.

3. Mao K, Xu X, Yang J, Yuan F, Gao X, Zhao J. Effects of small and large molecule emulsifiers on the characteristics of $\beta$-carotene nanoemulsions prepared by high pressure homogenization. Food Technol Biotechnol. 2009;47(3):336-42.

4. Gerster H. Anticarcinogenic effect of common carotenoids. Int J Vitamin and Nutr Res. 1993;63(2):93-121.

5. Lintig JV. Colours with functions: Elucidating the biochemical and molecular basis of carotenoid metabolism. In Annual Rev of Nutr. 2010;30:35-56.

6. Ribeiroa S, Chua S, Ichikawab S, Nakajimaa M. Preparation of nanodispersions containing $\beta$-carotene by solvent displacement method. Food Hydrocolloids. 2008;22(1):12-7.

7. Liang R, Shoemaker F, Yang X, Zhong F, Huang Q. Stability and bioaccessibility of $\beta$-carotene in nanoemulsions stabilized by modified starches. J Agric Food Chem. 2013;61(6):1249-57. 
8. Deng $\mathrm{X}$, Zhang $\mathrm{N}$, Tang $\mathrm{CH}$. Soy protein isolate as a nano carrier for enhanced water dispersibility, stability and bioaccessibility of $\beta$-carotene. J Sci Food Agric. 2017;97(7):2230-7.

9. Ishimoto K, Miki S, Ohno A, Nakamura Y, Otani S, Nakamura M, et al. $\beta$-Carotene solid dispersion prepared by hot-melt technology improves its solubility in water. J Food Sci Technol. 2019;56(7):3540-6.

10. Semalty A, Semalty M, Singh D, Rawat MSM. Preparation and characterization of phospholipid complexes of naringenin for effective drug delivery. J Incl Phenom Macrocycl Chem. 2010;67(3-4):253-60.

11. Bhattacharyya S, Ahammed SM, Saha BP, Mukherjee PK. The gallic acidphospholipid complex improved the antioxidant potential of gallic acid by enhancing its bioavailability. AAPS Pharm Sci Tech. 2013;14(3):1025-33.

12. Zhang WL, Cui Y, Fan YQ, Yang JK, Liu P, et al. Bioavailability and foam cells permeability enhancement of salvianolic acid $B$ pellets based on drugphospholipids complex technique. Eur J Pharm Biopharm. 2012;83(1):76-86.

13. Bhattacharyya S, Majhi S, Saha BP, Mukherjee PK. Chlorogenic acid phospholipid complex improve protection against UVA induced oxidative stress. J Photochem Photobiol B Biol. 2014;130:293-8.

14. Han X, Zhang Z, Shen H, Zheng J, Zhang G. Comparison of structures, physicochemical properties and in vitro bioactivity between ferulic acid- $\beta$ cyclodextrin conjugate and the corresponding inclusion complex. Food Res Int. 2019;125:1-10.

15. Valentina V, Vincenza C, Barbara F, Domenico M, Giuseppe A, Barbara T, et al. Physicochemical characterization and antioxidant activity evaluation of idebenone/hydroxypropyl- $\beta$-cyclodextrin inclusion complex. Biomolecules. 2019;9(531):2-29.

16. Pichot R, Watson RL, Norton IT. Phospholipids at the interface: Current trends and challenges. Int J Mol Sci. 2013;14(6):11767-94.

17. Singh D, Rawat MSM, Semalty A, Semalty M. Chrysophanol-phospholipid complex, J Therm Anal Calorim. 2012;111(3):2069-77.

18. Lin SY, Kao YH. Solid particulates of $\beta$-cyclodextrin inclusion complexes directly prepared by spray-drying technique. Int J Pharm. 1989;56(3):249-59.

19. Ghosh A, Biswas S, Ghosh T. Preparation and evaluation of silymarin $\beta$-cyclodextrin molecular inclusion complexes. J Young Pharmacists. 2011;3(3):205-10.

20. Munyendo WLL, Zhang Z, Abbad S, Waddad AY, Lv H, Baraza LD, et al. Micelles of TPGS modified apigenin phospholipid complex for oral administration: Preparation, in vitro and in vivo evaluation. J Biomed Nanotech. 2013;9(12):2034-47.

21. Choudhary A, Rana A, Aggarwal G, Kumar V, Zakir F. Development and characterization of an atorvastatin solid dispersion formulation using skimmed milk for improved oral bioavailability. Acta Pharmaceutica Sinica B. 2012;2(4):421-8.

22. Tan Q, Liu S, Chen X, Wu M, Wang H, Yin H, et al. Design and evaluation of a novel evodiamine-phospholipid complex for improved oral bioavailability. AAPS PharmSciTech. 2012;13(2):534-47.

23. Telange DR, Patil AT, Pethe AM, Anand S, Fegade H, Dave VS. Formulation and characterization of an apigenin-phospholipid phytosome (APLC) for improved solubility, in vivo bioavailability and antioxidant potential. Eur J Pharm Sci. 2017;108:36-49.
24. Telange DR, Nirgulkar SB, Umekar MJ, Patil AT, Pethe AM, Bali NR. Enhanced transdermal permeation and anti-inflammatory potential of phospholipids complex-loaded matrix film of umbelliferone: Formulation development, physico-chemical and functional characterization. Eur J Pharm Sci. 2019;131:23-38.

25. Telange DR, Denge RP, Patil AT, Gupta SV, Dave VS. Pentaerythritol as an excipeints/solid dispersion carrier for improved solubility and permeability of ursodeoxycholic acid. J Excp Food Chem. 2018;9(3):80-95.

26. Maiti K, Murugan V, Saha BP, Mukherjee K, Mukherjee PK. Exploring the effect of hesperetin-HPSC complex: A novel drug delivery system on the in vitro release, thera-peutic efficacy and pharmacokinetics. AAPS Pharm Sci Tech. 2009;10(3):943-50.

27. Savjani K, Gajjar A, Savjani J. Drug solubility: Importance and enhancement techniques. ISRN Pharm. 2012;1-10.

28. DePaz E, Martin A, Bartolome A, Largo M, Cocero MJ. Development of water soluble $\beta$-carotene formulations by high temperature, high-pressure emulsification and antisolvent precipitation. Food Hydrocolloids. 2014;37:14-24.

29. Semalty A, Tanwar Y, Semalty M. Preparation and characterization of cyclodextrin inclusion complex of naringenin and critical comparison with phospholipid complexation for improving solubility and dissolution. J Therm Anal Calorim. 2014;115(3):2471-78.

30. Zhang H, Sun X, Peng Q, Shi S, Gong T, Zhang J. Preparation, characterization and in vivo evaluation of a self-nanoemulsifying drug delivery system (SNEDDS) loaded with morin-phospholipid complex. Int J Nanomedicine. 2011;6:3405-14.

31. Savic R, Luo L, Eisenberg A, Maysinger D. Micellar nanocontainers distribute to defined cytoplasmic organelles. Science. 2003;300(5619):615-8.

32. Hadian Z, Maleki M, Abdi K, Atyabi F, Mohammadi A, Khaksar R. Preparation and characterization of nanoparticle $\beta$-cyclodextrin: Geraniol inclusion complexes. Iranian J Pharm Res. 2018;17(1):39-51.

33. Kaur P, Ghoshal $G$, Jain A. Bio-utilization of fruits and vegetables waste to produce $\beta$-carotene in solid state fermentation: Characterization and antioxidant activity. Process Biochem. 2019;76:155-64.

34. Lira MCB, Ferraz MS, DaSilva C, Cortes ME, Teixeira KI, Caetano NP, et al. Inclusion complex of usnic acid with $\beta$-cyclodextrin: Characterization and nanoencapsulation into liposomes. J Incl Phenom Macrocycl Chem. 2009;64(3-4):215-24.

35. Cai X, Luan Y, Jiang Y, Song A, Shao W, Li Z, et al. Huperzine A-phospholipid complex-loaded biodegradable thermosensitive polymer gel for controlled drug release. Int J Pharm. 2012;433(1-2):102-11.

36. Lasonder E, Weringa WD. An NMR and DSC study of the interaction of phospholipid vesicles with some anti-inflammatory agents. J Colloid Interface Sci. 1990;139(2):469-78.

37. Liu L, Guo QX. The driving forces in the inclusion complexation of cyclodextrins. J Incl Phenom Macroc Chem. 2002;42(1-2):1-14.

38. Rawat S, Jain SK. Enhancement of intestinal absorption of few cox-2 inhibitors through interaction with $\beta$-cyclodextrin. Indian J Pharm Sci. 2007;69(4):529-34.

39. Perrut M, Jung J, Leboeuf F. Enhancement of dissolution rate of poorlysoluble active ingredients by supercritical fluid processes Part I: Micronization of neat particles. Int J Pharm. 2005;288(1):3-10.

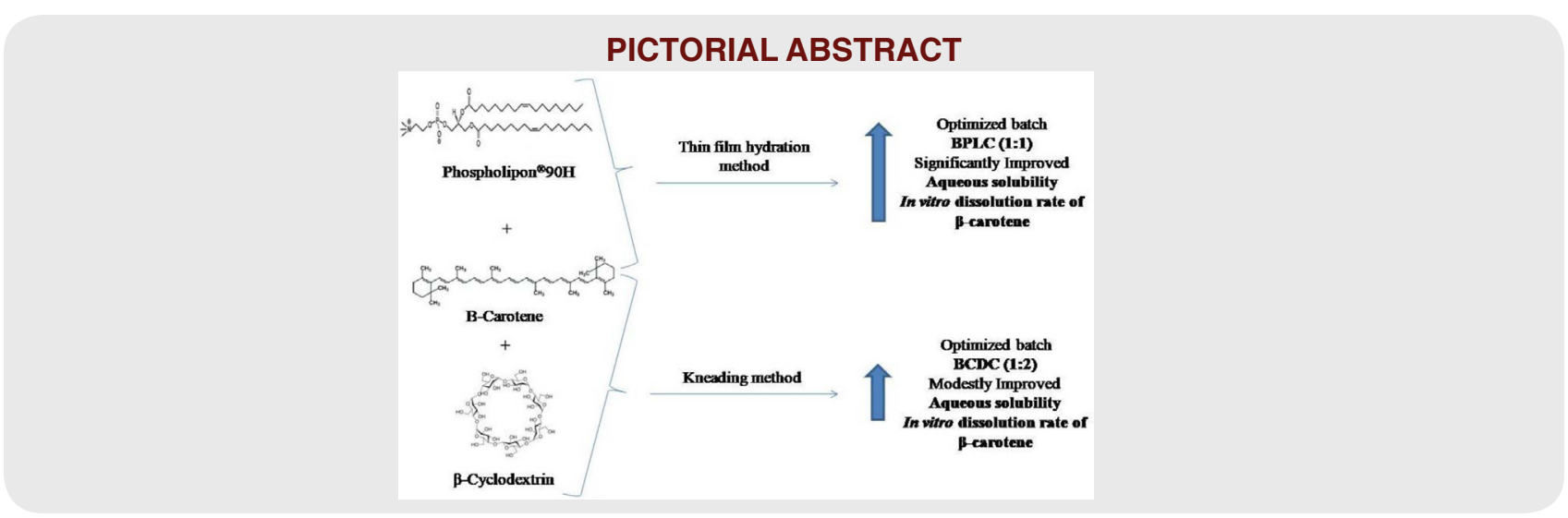




\section{SUMMARY}

Antioxidant is an important phytochemicals, substance and/or compound which significantly scavenge the free radicals and thereby, slow down the oxidation of biological macromolecules such as protein, lipids and carbohydrates. This activity is totally depend upon the biopharmaceutical, physico-chemical and functional properties of antioxidant compound. $\beta$-carotene, is one them, which is an essential part of carotenoid family and perform the same actions more appreciably. Moreover, it also provides an essential benefit to the human body via converting it into vitamin A. However, the poor aqueous solubility and low in vitro dissolution rate confined it become a strong candidate. In this study, the observed limitations of this wonder molecule were improved by preparing its formulations using Phospholipon ${ }^{\circledR} 90 \mathrm{H}$ and $\beta$-cyclodextrin carriers respectively. The prepared optimized formulations i.e. BPLC (1:1) and BCDC (1:2) were characterized using particle size and zeta potential, drug loading, complexation rate, FT-IR, DSC, solubility study and in vitro dissolution rate. Characterization studies demonstrated that optimized BPLC (1:1) were drastically improved an aqueous solubility and in vitro dissolution rate over to that of BCDC (1:2) and plain $\beta$-carotene. Therefore, the results of this study indicates that Phospholipon ${ }^{\circledR} 90 \mathrm{H}$ significantly improved the biopharmaceutical attributes compared to $\beta$-cyclodextrin and thus, can be used as promising carrier for the other compound with low aqueous solubility and in vitro dissolution rate.

\section{About Authors}

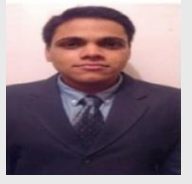

Mr.Saurabh Gujar, is working as research srtudent in Shobhaben Pratapbhai Patel School of Pharmacy and Technology management, SVKM's, NMIMS (Deemed to be University), Mumbai Campus, Mumbai,Maharashtra, India. His area interest is development of nanoformulations for BCS class II drugs.

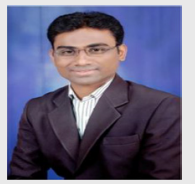

Dr. Darshan R. Telange, is working as Assistant Professor in Rajarshi Shahu College of Pharmacy, Buldhana, Maharashtra, India. According to research area, he is working on solid-state characterization, targeted nanoformulation and phytosomal formulations etc.

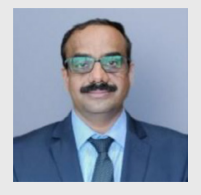

Dr. Anil M. Pethe, is working as Associate Dean at School of Pharamcy and Technology Management, SVKM's NMIMS (Deemed to be University), Hyderabad Campus, Telangana, India. His area of interest include development and evaluation of target based nanoformulations, QbD optimization and solubility enhancement etc.

Cite this article: Gujar S, Telange D, Pethe A. Supramolecular Complexes of Phospholipids and $\beta$-Cyclodextrin with Bioactive $\beta$-Carotene: A Comparative Physico-Chemical and Functional Evaluation. Indian J of Pharmaceutical Education and Research. 2020;54(2s):s220-s229. 